\title{
Dielectric properties of vapor-deposited propylbenzenes
}

Riechers, Birte; Guiseppi-Elie, A.; Ediger, Mark Donovan; Richert, Ranko

Published in:

Journal of Chemical Physics

DOI:

$10.1063 / 1.5125138$

Publication date:

2019

Document Version

Peer reviewed version

Citation for published version (APA):

Riechers, B., Guiseppi-Elie, A., Ediger, M. D., \& Richert, R. (2019). Dielectric properties of vapor-deposited propylbenzenes. Journal of Chemical Physics, 151(17). https://doi.org/10.1063/1.5125138

\section{General rights}

Copyright and moral rights for the publications made accessible in the public portal are retained by the authors and/or other copyright owners and it is a condition of accessing publications that users recognise and abide by the legal requirements associated with these rights.

- Users may download and print one copy of any publication from the public portal for the purpose of private study or research.

- You may not further distribute the material or use it for any profit-making activity or commercial gain.

- You may freely distribute the URL identifying the publication in the public portal.

Take down policy

If you believe that this document breaches copyright please contact rucforsk@kb.dk providing details, and we will remove access to the work immediately and investigate your claim. 


\title{
Dielectric properties of vapor-deposited propylbenzenes
}

\author{
Birte Riechers, ${ }^{1 *}$ A. Guiseppi-Elie, ${ }^{2}$ M. D. Ediger, ${ }^{3}$ and Ranko Richert ${ }^{1}$ \\ ${ }^{1}$ School of Molecular Sciences, Arizona State University, Tempe, Arizona 85287-1604, USA \\ ${ }^{2}$ Department of Biomedical Engineering, The Dwight Look College of Engineering, Texas A\&M University, \\ College Station, Texas 77843, USA \\ ${ }^{3}$ Department of Chemistry, University of Wisconsin-Madison, Madison, Wisconsin 53706, USA \\ *briecher@asu.edu
}

\begin{abstract}
Dielectric susceptibility data of vapor-deposited films of iso-propylbenzene (IPB) and npropylbenzene (NPB) have been recorded across a wide range of deposition temperatures, $T_{\mathrm{dep}}$, mostly below the glass transition temperature, $T_{\mathrm{g}}$. The results for the real and imaginary components of dielectric susceptibility are compared with recently published results for 2-methyltetrahydrofuran (MTHF). Common to all three systems are: (i) increased kinetic stability seen as higher onset temperature for the transformation to the liquid state for $T_{\mathrm{dep}} \approx 0.85 T_{\mathrm{g}}$, (ii) the reduction of the dielectric loss $\left(\chi^{\prime \prime}\right)$ for as-deposited glasses, a signature of increased packing density that is maximal for $T_{\mathrm{dep}} \approx 0.85 T_{\mathrm{g}}$, and (iii) a reduced level of the storage component $\left(\chi^{\prime}\right)$ for as-deposited glasses, an effect that is almost deposition temperature invariant for $T_{\mathrm{dep}}<T_{\mathrm{g}}$. Material specific behavior is observed when heating the as-deposited films to $1.2 T_{\mathrm{g}}$ : IPB and NPB transform directly into the ordinary liquid state if judged on the basis of dielectric susceptibility, whereas MTHF has been reported to enter an unusual liquid state prior to a liquid-liquid transition at higher temperatures. These results are discussed in the context of the curious scattering results reported by Ishii et al. for some benzene derivatives, which hint at a liquid-liquid transformation.
\end{abstract}

Keywords: glass transition, physical vapor deposition, kinetic stability, polyamorphism, dielectric susceptibility 


\section{Introduction}

Physical vapor deposition of glass forming materials has received considerable attention in recent years, because the properties of such films can differ substantially from the counterpart obtained by cooling the liquid..$^{1,2,3,4,5,6,7,8,9}$ Supercooled liquids are ordinarily obtained by lowering the temperature of a liquid from above the melting point $T_{\mathrm{m}}$ to below, at a rate at which crystallization can be avoided, typically $1-10 \mathrm{~K} \mathrm{~min}^{-1}$ for molecular glass-formers. Further cooling to temperatures below the glass transition temperature, $T_{\mathrm{g}}$, leads to a glassy state, and the properties in this non-equilibrium state depend on the cooling rate and the time spent at sub- $T_{\mathrm{g}}$ temperatures or aging. Physical vapor deposition (PVD) onto substrates with temperatures set to values not far below $T_{\mathrm{g}}$ also leads to a glassy state, but often with higher kinetic stability and density, and with lower enthalpy and entropy compared to the supercooled liquid. ${ }^{10}$ Apart from possible PVD induced anisotropy effects, the properties of asdeposited films are similar to what is expected from glasses prepared by ordinary cooling and after aging times of thousands or millions of years. ${ }^{5,11,12}$

The feature made responsible for the unusual and interesting properties of PVD films is the combination of a low temperature (and thus strong driving force towards low energy states) with the relatively high surface mobility, ${ }^{13}$ which allows newly arrived molecules to sample a large parameter space during deposition. ${ }^{10}$ As the local environment at the surface dominates the arrest of molecules for sufficiently low deposition rates, the difference between glasses obtained by vapor deposition and ordinary cooling may depend on the intermolecular interactions. This is reflected by the fact that many alcohols do not form stable glasses due to hydrogen bonding and the concomitantly small surface mobility. ${ }^{14,15}$

Recent experiments have revealed that the dielectric properties of as-deposited films also differ from their ordinary counterparts obtained by cooling. Some vapor-deposited polyalcohols such as glycerol have shown unusual dielectric behavior in their liquid state. ${ }^{16}$ Further examples are the reduced amplitudes of secondary relaxations in glassy films, believed to reflect denser packing in PVD films. ${ }^{17}$ In terms of the dependence on deposition temperature $\left(T_{\mathrm{dep}}\right)$ this reduction of the dielectric loss correlates with the extent of kinetic stability, both being most pronounced for $T_{\mathrm{dep}} \approx 0.85 T_{\mathrm{g}}$. For 2 methyltetrahydrofuran (MTHF), it has also been observed that as-deposited samples show very low dielectric constants in the glassy state $\left(\varepsilon_{\infty}\right)$, and a lowered static dielectric constant in the liquid state $\left(\varepsilon_{s}\right)$, a state that requires extensive annealing prior to recovering the ordinary liquid behavior. ${ }^{18}$ The latter feature of finding two distinct liquid states for MTHF suggests that PVD may be an effective 
route for the discovery of polyamorphism and liquid-liquid transitions. Indications for similar behavior in propylbenzenes have been reported by Ishii and collaborators on the basis of optical experiments, ${ }^{19}$ which provides a motivation for a dielectric investigation of these liquids.

In this context of polyamorphism of liquids obtained from vapor-deposited samples, the earlier studies by Ishii et al. can be interpreted as an alternative liquid state that appears upon heating a film deposited at temperatures below $0.7 T_{\mathrm{g}}{ }^{19,20,21,22}$ This has been reported for ethylbenzene, $n$ propylbenzene, and iso-propylbenzene (cumene), and the signature of the unusual state is excessive scattering for a certain temperature range in the supercooled liquid state observed in optical reflectivity experiments. A further increase in the temperature appears to recover the ordinary liquid before crystallization sets in at the melting temperature.

In the present study, vapor-deposited films of two propylbenzenes were characterized by dielectric techniques and compared with the recently published results obtained for MTHF. ${ }^{18}$ Common features are the suppression of the real part of the dielectric susceptibility $\left(\chi^{\prime}\right)$ for the as-deposited glasses, an effect that is independent of the deposition temperature, $T_{\mathrm{dep}}$, as long as $T_{\mathrm{dep}}<T_{\mathrm{g}}$. Furthermore, all samples show an enhanced kinetic stability and reduced values of the loss $\left(\chi^{\prime \prime}\right)$ for values of $T_{\text {dep }}$ near $0.85 T_{\mathrm{g}}$. Unlike MTHF, however, both propylbenzenes display dielectric losses below $T_{\mathrm{g}}$ that exceed the level of the ordinary glass when the deposition temperature is below $0.6 T_{\mathrm{g}}$, indicative of a lower than ordinary packing density. Another difference to the MTHF behavior is the observation that the propylbenzenes recover the ordinary liquid and glassy states via annealing just above $T_{\mathrm{g}}$, i.e., without indication of polyamorphism resulting from the PVD preparation.

\section{Experimental}

The compounds 2-methyltetrahydrofuran (MTHF, 99+\%, stabilizer-free, Acros Organics), isopropylbenzene (IPB, 99.9\%, Acros Organics), and n-propylbenzene (NPB, >99.0\%, Sigma-Aldrich) were used as received and vapor-deposited films were prepared using a custom-designed setup. ${ }^{17,18} \mathrm{~A}$ microlithographically fabricated (lift off) dual-sensing chip (Model IME 1050.5-FD-Au, ABTECH Scientific, Inc.) with 50 pairs of interdigitated electrodes (IDE) per sensor on top of a $0.5 \mathrm{~mm}$ thick borosilicate glass served as substrate. ${ }^{23}$ Each digit had a width of $10 \mu \mathrm{m}$, a length of $5 \mathrm{~mm}$, and was separated by $10 \mu \mathrm{m}$ from its neighbors. The electrodes on the chips were contacted via bonding pads using a custom-built spring-loaded connector. ${ }^{18}$

The chip was pressed onto a brass holder that was mounted onto the cold-stage of a closed-cycle helium cryostat (Leybold RDK 10-320). The temperature was controlled by a Lakeshore Model 340 
using DT-470-CU sensors, facilitating temperature adjustments between $30 \mathrm{~K}$ and $300 \mathrm{~K}$. Temperatures given in the following are based on measurements by a sensor located at the brass block that held the sensor chip. To correct for the temperature difference originating from thermal lag (typically in the order of $2 \mathrm{~K}$ to $5 \mathrm{~K}$ ), all data of each sample were shifted uniformly so that the loss peak of the experimental data after annealing coincided with the loss peak position of reference data obtained for liquid-cooled (bulk) material measured in a standard parallel-plate capacitor, see Fig. S1, Fig. S2, and Fig. S3 in Supplementary Material (SM). ${ }^{24}$

In order to determine the geometric capacitance, $C_{\text {geo, }}$, of each of the two sensors on the chip, 2ethyl-1-hexanol was used for calibration. ${ }^{18}$ For a capacitor that is completely filled with material, the permittivity $\varepsilon^{*}$ on each side (deposition or substrate side) is determined by the ratio of capacitances using $\varepsilon^{*}=C^{*} / C^{*}$ geo. For annealed samples thinner than half of the assumed field height $H,{ }^{25}$ the relation that connects the incremental capacitance, $\Delta C$, to the observed susceptibility, $\chi$ obs, and the film thickness, $d$, is given by

$$
\Delta C=C_{g e o} \chi_{o b s}^{\prime}(T)=C_{g e o} \chi_{r e f}^{\prime}(T) \times \frac{d}{H},
$$

where $\chi_{\text {ref }}{ }^{*}(T)=\varepsilon_{\text {ref }}{ }^{*}(T)-1$ denotes reference data. ${ }^{24}$ Capacitances were recorded in the frequency range from $100 \mathrm{~Hz}$ to $20 \mathrm{kHz}$ using an Andeen-Hagerling ultra-precision bridge (AH-2700A) connected to one of the two IDEs that are located on the chip.

After deposition at a specific temperature, $T_{\text {dep }}$, the sample was cooled down to the initial temperature of the first scan, $T_{\text {ini. }}$. For each sample, three successive temperature scans were performed by increasing the temperature in steps of $1.5 \mathrm{~K}$ in case of MTHF and $1 \mathrm{~K}$ for IPB and NPB. After the temperature leveled within $0.2 \mathrm{~K}$ of the set temperature value for $45 \mathrm{~s}$, dielectric measurements at 8 frequencies between $100 \mathrm{~Hz}$ and $20 \mathrm{kHz}$ were conducted prior to approaching the next temperature. This is equivalent to an average heating rate of about $1 \mathrm{~K} \mathrm{~min}^{-1}$. After reaching the final temperature of the first temperature scan, the sample was cooled back down to $T_{\text {ini }}$ and kept at that temperature for 10 min. The same procedure was repeated for the second and third scans, but with the final temperature of the third scan equal to $295 \mathrm{~K}$ to induce desorption of the sample material from the interdigitated electrode device (IDE).

To correct for the contribution of the borosilicate substrate to the overall capacitance, a temperature scan of the pristine chip was conducted with the same thermal protocol as described for the third temperature scan. After subtracting this contribution from data measured at a frequency $v=1 \mathrm{kHz}$, the resulting values of loss and storage susceptibility, $\chi_{\exp }^{\prime}(T)$ and $\chi^{\prime \prime} \exp (T)$, reflecting the material's 
response were normalized by dividing data of all three temperature scans by $a=\chi^{\prime} 3 \mathrm{rd} \operatorname{scan}\left(T_{\mathrm{ref}}\right) / \chi^{\prime}$ ref $\left(T_{\text {ref }}\right)$. This assumes that the annealing during the first and second scan leads to a glass whose $\chi^{\prime}$ value at $T_{\text {ref }}$ is virtually identical to the reference obtained by cooling the bulk liquid. The reference temperature used for the normalization of MTHF-data was set to $T_{\mathrm{ref}}{ }^{\mathrm{MTHF}}=52.5 \mathrm{~K}$. For IPB and NPB, the reference temperatures were $T_{\mathrm{ref}}^{\mathrm{IPB}}=136 \mathrm{~K}$ and $T_{\mathrm{ref}}^{\mathrm{NPB}}=130 \mathrm{~K}$. By using the relation $d=a H$, the film thickness of the deposited samples was determined and, aided by the duration of the deposition, $t_{\text {dep }}$, the deposition rate, $r_{\mathrm{dep}}=d / t_{\mathrm{dep}}$, was deduced.

In the following, vapor-deposited films are presented that were prepared at $T_{\text {dep }}$ from $0.3 T_{\mathrm{g}}$ to $1.1 T_{\mathrm{g}}$ with $r_{\text {dep }}$ of about $0.2 \mathrm{~nm} \mathrm{~s}^{-1}$, and resulting $d$ between $450 \mathrm{~nm}$ and $1 \mu \mathrm{m}$. The notation $T_{\mathrm{g}}$ refers to the caloric glass transition temperature, which correspond to $T_{\mathrm{g}}{ }^{\mathrm{MTHF}}=91 \mathrm{~K},{ }^{26,27} T_{\mathrm{g}}{ }^{\mathrm{IPB}}=129 \mathrm{~K},{ }^{19}$ and $T_{\mathrm{g}}{ }^{\mathrm{NPB}}$ $=126 \mathrm{~K} \cdot{ }^{19}$ The data for MTHF are the results of experiments conducted in the course of a previous study, ${ }^{18}$ but this earlier publication does not show all the MTHF data presented here.

\section{Results}

The loss component of the dielectric susceptibility, $\chi^{\prime \prime}$, is plotted against temperature $T$ for MTHF, IPB, and NPB in Fig. 1. For each of the three materials, curves for as-deposited ('AD') samples for different deposition temperatures, $T_{\text {dep }}$, are shown, together with the behavior obtained after annealing ('ann') at temperatures up to $1.2 T_{\mathrm{g}}$, i.e., data obtained during the second or third temperature scan. Common features of these three systems are the suppressed amplitudes of the dielectric susceptibility for as-deposited samples, $\chi^{\prime \prime} \mathrm{AD}$, observed for temperatures $T<T_{\mathrm{g}}$ relative to the loss component, $\chi^{\prime \prime}$ ann, of samples after annealing. This suppression is strongly correlated with $T_{\mathrm{dep}}$, and is most intense for deposition temperatures near $T_{\mathrm{dep}}=0.85 T_{\mathrm{g}}$. Furthermore, upon heating, $\chi^{\prime \prime} \mathrm{AD}$ curves reveal a delayed transformation from the as-deposited to the supercooled liquid state for samples deposited in this temperature range. These observations were previously discussed in connection to a more dense packing, ${ }^{18,28,29}$ resulting in the suppression of secondary relaxations ${ }^{17,30}$ and enhanced kinetic stability in terms of a delayed onset of the primary relaxation as characteristic features of ultrastable glasses. ${ }^{11}$ Consistent with earlier dielectric studies, ${ }^{18,17}$ the suppression of the loss component of the dielectric constant is used as an indicator for the degree of stability, comparable to the delay of the transformation onset temperature of the stable state to the supercooled liquid.

Within the temperature scan of as-deposited MTHF films, a distinct increase in the signal amplitude is observed in the range $T=0.9 T_{\mathrm{g}}$ to $T=1.0 T_{\mathrm{g}}$ for films deposited at temperatures below $T_{\mathrm{dep}}=0.8 T_{\mathrm{g}}$ or above $T_{\text {dep }}=0.9 T_{\mathrm{g}}$. This increase manifests itself in Fig. 1 in form of a bump or a shoulder in case of 
MTHF and is well separated from the onset of the transformation, which occurs at temperatures $T=$ $1.0 T \mathrm{~g}$ to $T=1.1 T_{\mathrm{g}}$. Similarly, IPB and NPB display broad maxima in the same range of the temperature scan and for the same deposition temperature conditions, $T_{\text {dep }}<0.8 T_{\mathrm{g}}$ or $T_{\mathrm{dep}}>0.9 T_{\mathrm{g}}$.

In order to better illustrate the changes in $\chi^{\prime \prime} \mathrm{AD}(T)$ relative to $\chi^{\prime \prime}$ ann $(T)$ for the three compounds, Fig. 2 depicts the loss ratio of as-deposited and annealed data, $\chi^{\prime \prime}{ }_{\mathrm{AD}} / \chi^{\prime \prime}$ ann. In the case of MTHF, this ratio remains below unity, i.e., $\chi^{\prime \prime} \mathrm{AD}(T)$ is always below the loss $\chi^{\prime \prime} \mathrm{ann}(T)$ of the annealed material for all values of $T_{\text {dep }}$. While the level of the loss ratio depends on $T_{\text {dep }}$, it varies only marginally for a given sample for temperatures up to $0.9 T_{\mathrm{g}}$, where the shoulder observed in $\chi^{\prime \prime} \mathrm{AD}(T)$ manifests itself in form of a slight maximum in the loss ratio. Only samples deposited in the range $0.8 T_{\mathrm{g}}<T_{\mathrm{dep}}<0.9 T_{\mathrm{g}}$ do not show this maximum. The minimum at $T>T_{\mathrm{g}}$ within the temperature scan is more intense for films with stronger kinetic stability and more intense suppression of relaxational contributions in the as-deposited state compared to the annealed sample.

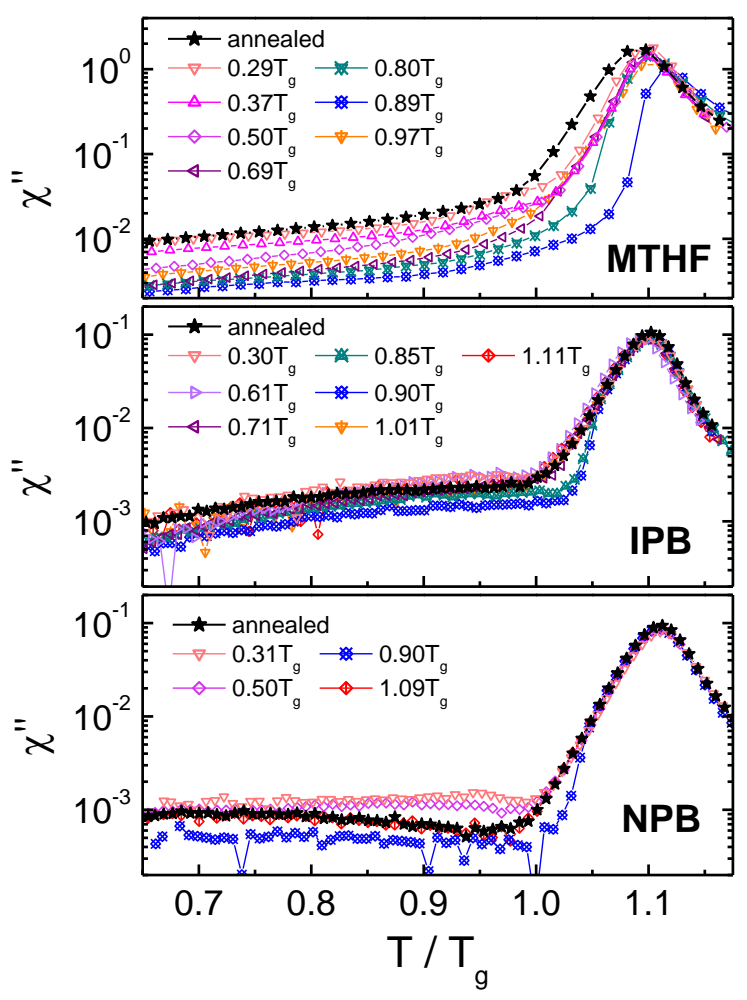

FIG. 1. Experimental results for the loss component of the dielectric susceptibility, $\chi^{\prime \prime}$ at $v=1 \mathrm{kHz}$, of asdeposited MTHF, IPB, and NPB, plotted against reduced temperature, $T / T_{\mathrm{g}}$, for various deposition temperatures, $T_{\text {dep. }}$. Deposition rates vary between $0.1 \mathrm{~nm} \mathrm{~s}^{-1}$ to $0.2 \mathrm{~nm} \mathrm{~s}^{-1}$ and sample thicknesses are between $450 \mathrm{~nm}$ and 1000 $\mathrm{nm}$. Data of samples after annealing at temperatures up to $1.2 T_{\mathrm{g}}$ are included for comparison as black stars.

In contrast to MTHF, the amplitudes for $\chi^{\prime \prime}$ in the glass show enhanced values for IPB and NPB compared to the annealed material for low deposition temperatures, $T_{\mathrm{dep}} \leq 0.61 T_{\mathrm{g}}$. This behavior is 
observed already in Fig. 1, but more clearly visible in Fig. 2 for the propylbenzenes. Here, values above unity occur in the range of $0.9 T_{\mathrm{g}}<T<1.0 T_{\mathrm{g}}$ for IPB and observed over an even broader range, $0.8 T_{\mathrm{g}}<$ $T<1.0 T_{\mathrm{g}}$, for NPB. For a sample of IPB deposited at $0.71 T_{\mathrm{g}}$, only a slight maximum with a loss ratio just below unity is observed near $T / T_{\mathrm{g}}=0.97$ (cf. IPB panel of Fig. 2), comparable to the behavior of MTHF. Fig. 2 reiterates that deposition temperatures in the range $0.8 T_{\mathrm{g}}<T_{\text {dep }}<0.9 T_{\mathrm{g}}$ lead to strongly suppressed loss amplitudes for all three materials. Along with the strongest $\chi^{\prime \prime}$-suppression, a delay of the transformation from the as-deposited to the supercooled liquid state towards higher temperatures is again observed, but this increased onset temperature leads to a dip in the quantity $\chi^{\prime \prime} \mathrm{AD} / \chi^{\prime \prime}$ ann. For $T_{\mathrm{dep}}$ values close to $T_{\mathrm{g}}$, the curves for the propylbenzenes resemble the behavior of the annealed material, which becomes evident by a loss-ratio close to unity.

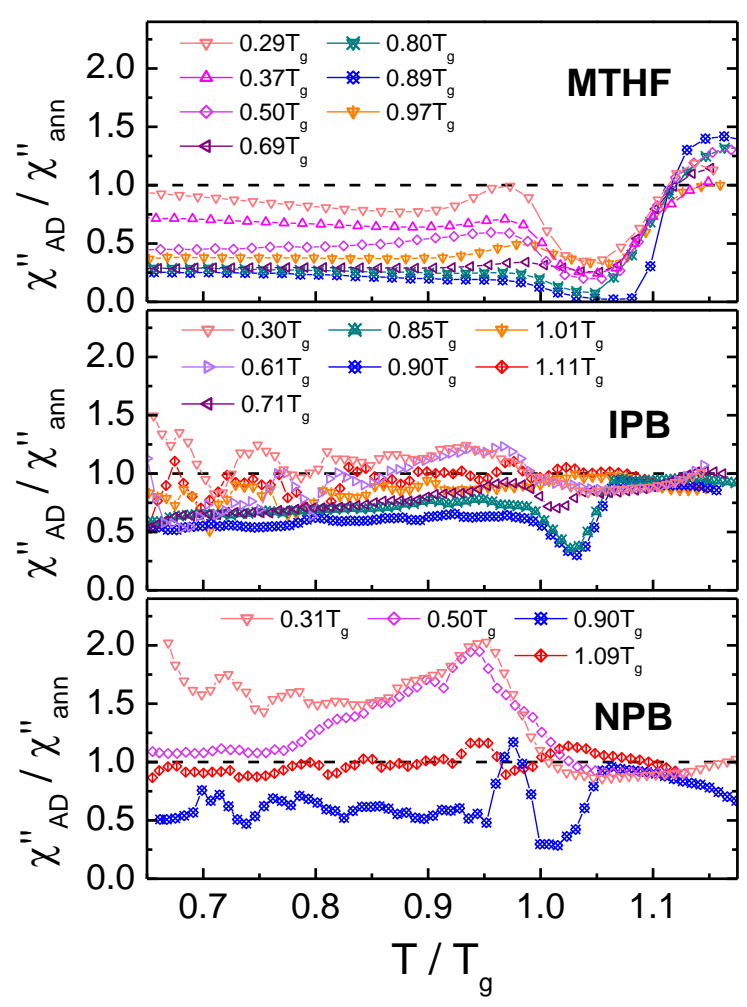

FIG. 2. Experimental results for the loss ratio of as-deposited to annealed dielectric loss, $\chi^{\prime \prime}{ }_{\mathrm{AD}} / \chi^{\prime \prime}$ ann at $v=1 \mathrm{kHz}$, of MTHF, IPB, and NPB, plotted against reduced temperature, $T / T_{\mathrm{g}}$, for various deposition temperatures, $T_{\text {dep }}$. Propylbenzene data were smoothed by sliding averages over six data points, see SM for original data.

The storage component of the dielectric susceptibility, $\chi^{\prime}$, is plotted against temperature for MTHF, IPB, and NPB in Fig. 3. Each frame includes data of as-deposited samples for a variety of deposition temperatures, data of the material annealed at temperatures up to $1.2 T_{\mathrm{g}}$, and reference data associated with the ordinary bulk liquid measured in a standard capacitor. For MTHF two distinct properties of asdeposited films are obvious that differ from reference data. One is the suppression of the storage 
contribution of as-deposited films relative to the annealed counterpart for temperatures below the glass transition, $T<T \mathrm{~g}$. The other is the suppression of the $\chi^{\prime}$ amplitude in the liquid state, which recovers the level of the reference data only after annealing at higher temperatures, $T>1.3 T_{\mathrm{g}}$. This change in signal amplitude in the liquid state of MTHF amounts to a factor of about two and has been discussed in terms of a liquid-liquid phase transition. ${ }^{18}$

For IPB and NPB, the $\chi^{\prime}$ curves in Fig. 3 show the suppression of $\chi^{\prime}$ AD only prior to annealing, while a temperature excursion to $1.2 T_{\mathrm{g}}$ recovers the values of the liquid-cooled material (reference). The suppression of $\chi^{\prime} \mathrm{AD}$ at low temperatures is less strongly developed than in the case of MTHF.

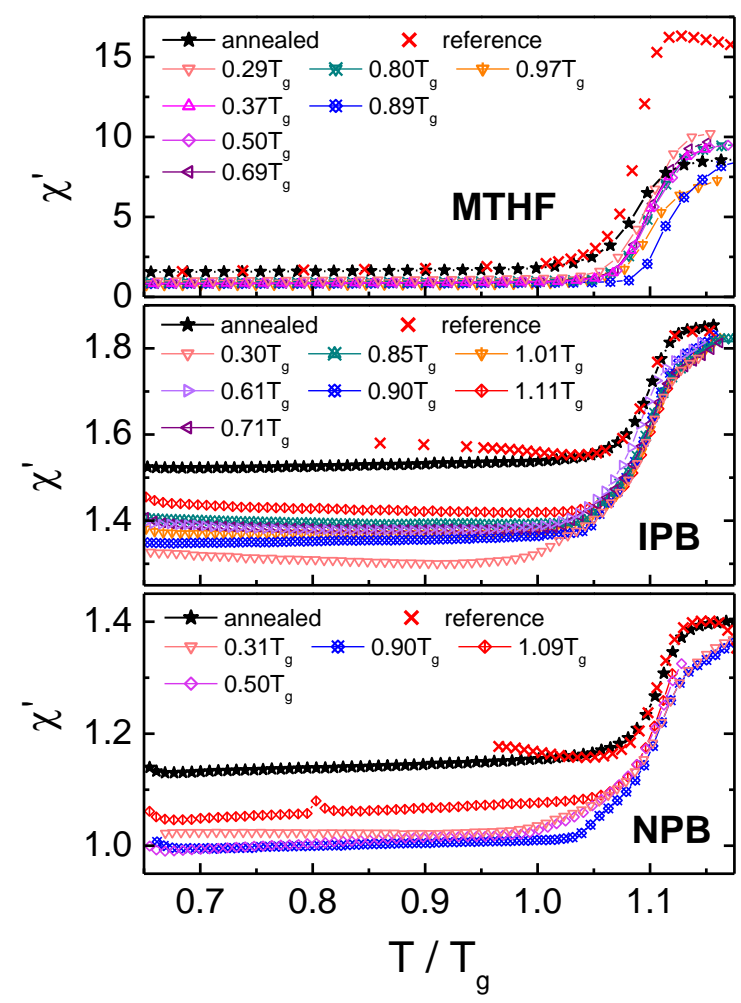

FIG. 3. Experimental results for the storage component of the dielectric susceptibility, $\chi^{\prime}$ at $v=1 \mathrm{kHz}$, of asdeposited MTHF, IPB, and NPB, plotted against reduced temperature, $T / T_{\mathrm{g}}$, for various deposition temperatures, $T_{\text {dep }}$. Data of samples after annealing at temperatures up to $1.2 T_{\mathrm{g}}$ (black stars) and those of liquid-cooled material (red crosses) are included for comparison.

As a measure for the degree of suppression in the glassy state at $T=0.75 T_{\mathrm{g}}$, the storage component ratio, $\chi^{\prime} \mathrm{AD} / \chi^{\prime}$ ann , is plotted in Fig. 4. For MTHF, a systematic variation of $\chi^{\prime} \mathrm{AD} / \chi^{\prime}$ ann with film thickness $d$ is observed, which amounts to an increase by about $20 \%$ over the range of experimental film thicknesses, see Fig. 4a. In the case of IPB, however, a correlation between $\chi^{\prime} \mathrm{AD} / \chi^{\prime}$ ann and $d$ is not evident. Instead, the storage ratio for IBP increases with $T_{\text {dep }}$ by about $7 \%$, see Fig. $4 \mathrm{~b}$. Clearly, the suppression effect for MTHF exceeds that of IPB for all $d$ and $T_{\text {dep }}$ conditions. The $\chi^{\prime}$ AD $/ \chi^{\prime}$ ann values for 
NPB are all near 0.90, i.e., similar to those of IPB. However, data for NPB are not shown as they are too scattered to reveal meaningful trends with the variation of $d$ or $T_{\text {dep. }}$.
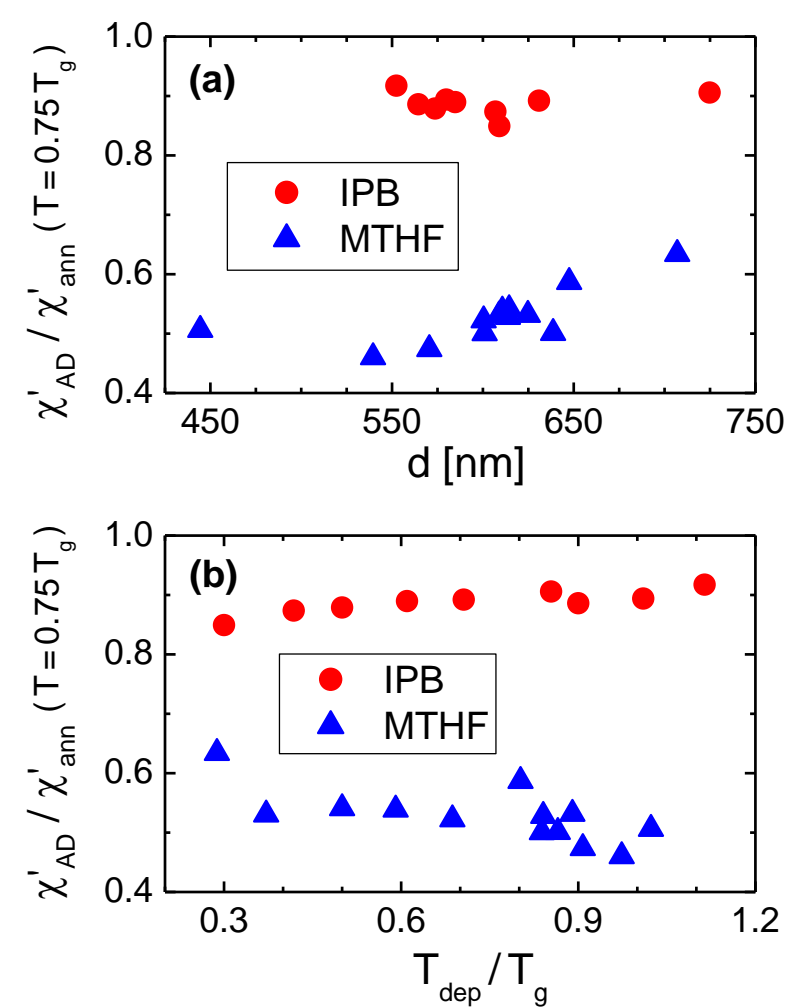

FIG. 4. Storage ratio, $\chi_{\mathrm{AD}}^{\prime} / \chi_{\text {ann }}^{\prime}$, of MTHF and IPB plotted against (a) film thickness $d$, and (b) reduced deposition temperature $T_{\mathrm{dep}} / T_{\mathrm{g}}$. Values are based on data of Fig. 3 , with the ratios determined for $T=0.75 T_{\mathrm{g}}$.

\section{Discussion}

Physical vapor deposition is an established tool for the preparation of samples in the ultrastable state and allows for tailoring material properties by controlling deposition conditions, such as the deposition rate, $r_{\text {dep }}$, and deposition temperature, $T_{\text {dep. }}$. Since the study on the formation of ultrastable films of indomethacin and a benzene derivative by Swallen et al., ${ }^{1}$ numerous materials have been reported to form states of ultrastable behavior after deposition at substrate temperatures in the range $0.80 T_{\mathrm{g}}<T_{\mathrm{dep}}<0.90 T_{\mathrm{g}}$ at deposition rates of $1 \mathrm{~nm} \mathrm{~s}^{-1}$ or less. ${ }^{10}$

At substrate temperatures near $T_{\mathrm{dep}}=0.85 T_{\mathrm{g}}$, molecules within the most recently deposited layers of the samples display mobilities that are much higher than in the bulk state at the same low temperature. This allows for a more efficient sampling of configurational space during the deposition process, while the low temperature provides a driving force towards low enthalpy and densely packed states. Therefore, a suitable choice of deposition temperature and rate facilitates the fabrication of materials 
with lower enthalpies and entropies than obtainable by conventional cooling of the liquid. By contrast, the driving force towards a more stable state is reduced at deposition temperatures in excess of $T_{\mathrm{dep}}=$ $0.90 T_{\mathrm{g}}$, and even the bulk material may be able to age within the experimental time scale. Thus, $T_{\mathrm{dep}} \approx$ $T_{\mathrm{g}}$ results in a state not far from the equilibrium state obtained by ordinary cooling. For deposition temperatures sufficiently below $T_{\text {dep }}=0.80 T_{\mathrm{g}}$, the surface mobility of freshly deposited molecules is too low for significant equilibration, and molecules can become trapped relatively high within the potential energy landscape (as in a rapid quench). This results in the deposition of a less stable and less dense state, even for low deposition rates and particularly for materials with strong intermolecular interactions that further inhibit surface mobility.

In this study we report dielectric data on MTHF, IPB, and NPB that were deposited in a wide range of deposition temperatures for deposition rates well below $1 \mathrm{~nm} \mathrm{~s}^{-1}$. Section IV.A. discusses the stability of as-deposited films and their transformation upon heating, as revealed by analyzing the loss component, $\chi^{\prime \prime}$, of the dielectric susceptibility. Section IV.B. discusses how deposition conditions impact the dielectric storage component, $\chi^{\prime}$. Finally, Section IV.C. focuses on signatures of polyamorphism and possible liquid-liquid transitions in these vapor-deposited samples.

\section{A. Stability and transformation of as-deposited films}

Samples of MTHF, IPB, and NPB that were deposited at temperatures between $T_{\mathrm{dep}}=0.80 T_{\mathrm{g}}$ and $T_{\text {dep }}=0.90 T_{\mathrm{g}}$ show clear signatures of ultrastable behavior: a delay of the onset temperature of transformation of the as-deposited material to the supercooled liquid state compared with the caloric glass transition temperature $T_{\mathrm{g}}$, as well as the suppression of the loss contribution to dielectric permittivity of the as-deposited glass. The latter effect is shown in Fig. 5 as the ratio of loss values for the as-deposited and annealed case, $\chi^{\prime \prime} \mathrm{AD} / \chi^{\prime \prime}$ ann versus $T_{\text {dep }}$, with the loss values evaluated at a temperature of $T=0.85 T \mathrm{~g}$. For all three systems of this study, Fig. 5 reveals that the suppression of loss in the glassy state is most pronounced near $T_{\mathrm{dep}}=0.85 T_{\mathrm{g}}$, underlining the connection between this suppression of residual mobility in the glass and kinetic stability. These observations are comparable to what has been reported for numerous materials. ${ }^{11,17,18,28,29,30,31,32,33,34}$ They are linked to an enhanced kinetic stability and an increased packing density relative to the glass formed by cooling a liquid below $T_{\mathrm{g}}$, which are the hallmarks of the ultrastable state obtained by physical vapor deposition.

In light of the increased packing density in films with high kinetic stability, the suppression of $\chi^{\prime \prime} \mathrm{AD}$ is understood as a restriction of molecular reorientations of the secondary or Johari-Goldstein (JG) $\beta$ process in the glassy state. ${ }^{17,35}$ Based on NMR-experiments performed on toluene, the JG $\beta$-process can 
be described by reorientational processes within cone angles between $2^{\circ}$ and $10^{\circ}$ for $90 \%$ of the molecules. ${ }^{36,37}$ The suppression of the loss-amplitude in the glass can be explained either in terms of an elimination of the larger cone angles or by a uniform limitation of the amplitude of reorientational processes. ${ }^{17}$ The residual signal consists of nearly constant loss (NCL) dynamics and a remaining contribution of the JG $\beta$-process.

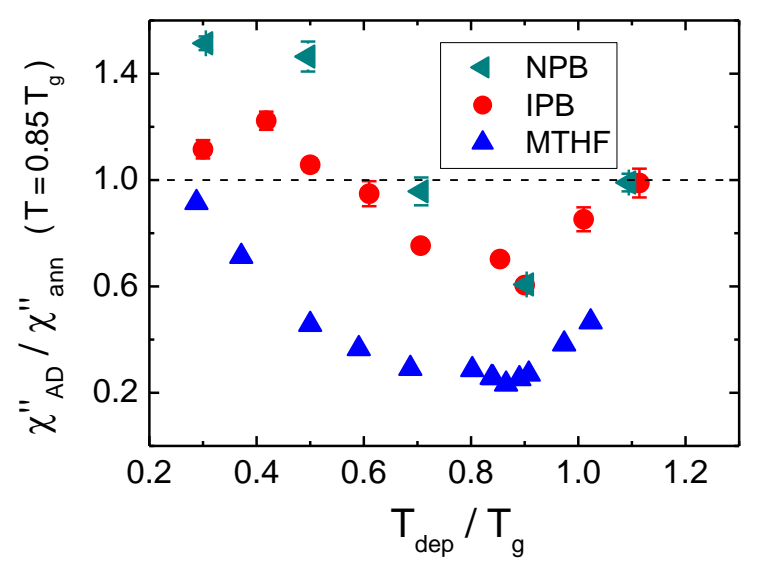

Fig. 5. Loss ratio, $\chi^{\prime \prime}{ }_{\mathrm{AD}} / \chi^{\prime \prime}$ ann, of MTHF, IPB, and NPB plotted against reduced deposition temperature, $T_{\mathrm{dep}} / T_{\mathrm{g}}$. The ratio was determined for a temperature of $T=0.85 T_{\mathrm{g}}$ based on data of Fig. 2 .

The kinetic stability of vapor-deposited samples indicates that the primary structural $(\alpha-)$ relaxation process is strongly inhibited for temperatures below the transition towards the supercooled liquid. This is seen most clearly in Fig. 1 for the $0.80 T_{\mathrm{g}}<T_{\mathrm{dep}}<0.90 T_{\mathrm{g}}$ cases, where the signature of kinetic stability is the steep rise of $\chi^{\prime \prime}(T)$ with onset at $T>T_{\mathrm{g}}$ and delayed merging into the curve representing the annealed sample. For some curves associated with lower kinetic stability, i.e., those with deposition temperatures further away from $T_{\mathrm{dep}} \approx 0.85 T_{\mathrm{g}}$, a moderate rise in $\chi^{\prime \prime}(T)$ can be observed starting already in the $0.8 T_{\mathrm{g}}<T<1.0 T_{\mathrm{g}}$ range, see Fig. 1 or Fig. 2 . The origin of this behavior is addressed in what follows.

For deposition temperatures outside the range $0.80 T_{\mathrm{g}}<T_{\mathrm{dep}}<0.90 T_{\mathrm{g}}$, as-deposited samples can still show a residual delay in the onset temperature of the transformation to the supercooled state (cf. Fig. 1, Fig. 2, and Fig. 3) and some suppression of secondary relaxations (cf. Fig. 1 and Fig. 2). This is observed not only in this study but also in numerous previous works on ultrastable glasses. ${ }^{10}$ In these situations, the molecular packing is less dense and thus affects the secondary process less intensely compared with the $T_{\mathrm{dep}} \approx 0.85 T_{\mathrm{g}}$ situation. As a result, such deposition conditions lead to a distinct separation of the primary and secondary processes, where the amplitude of the $\beta$ process is still resolved and the $\alpha$-process onset is shifted upward along the temperature scale. ${ }^{38}$ The delayed onset of 
the $\alpha$-process translates into the absence of cage-breaking, so that the $\beta$-relaxation appears in terms of its intrinsic spectrum, i.e., without merging with the $\alpha$-process. This explains the shoulder or hump observed in the $0.9 T_{\mathrm{g}}<T<1.0 T_{\mathrm{g}}$ range of those MTHF curves in Fig. 1 that are associated with deposition temperatures $T_{\text {dep }}<0.8 T_{\mathrm{g}}$ or $T_{\text {dep }}>0.9 T_{\mathrm{g}}$. In Fig. 2 , these signatures of the secondary relaxation appear as a broad peak in the temperature range of $0.90 T_{\mathrm{g}}<T<1.00 T_{\mathrm{g}}$. In support of this interpretation, Fig. 6 shows the susceptibility of bulk MTHF at $v=1 \mathrm{kHz}$, where open symbols reflect the loss after subtracting the $\alpha$-process by power law extrapolation on the high frequency side of the $\varepsilon^{\prime \prime}(\omega)$ spectra. The temperature profile of the $\varepsilon^{\prime \prime}{ }_{\beta, \text { only }}$ curve in Fig. 6 resembles the shoulders in Fig. 1 just below $T_{\mathrm{g}}$. Analogous effects can be seen for the propylbenzenes, IPB and NPB, in Fig. 1 and Fig. 2. As a consequence of the above considerations, the rise of $\chi^{\prime \prime}$ for $T<T_{\mathrm{g}}$ is not understood as an early onset of transformation behavior, but rather a signature of the secondary relaxation processes in the glassy state, made visible via the separation of $\alpha$ - and $\beta$-contributions to the loss.

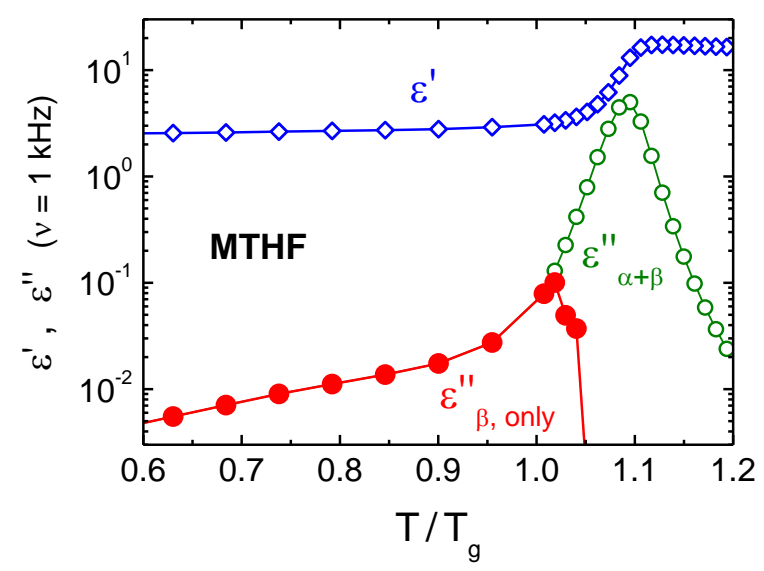

Fig. 6. Dielectric permittivity data, $\varepsilon^{\prime}$ and $\varepsilon^{\prime \prime}$, for MTHF at $v=1 \mathrm{kHz}$. The storage component $\varepsilon^{\prime}$ is shown as blue open diamonds, the loss $\varepsilon^{\prime \prime}$ is shown as green open circles and designated $\varepsilon^{\prime \prime}{ }_{\alpha+\beta}$. Red closed symbols labeled $\varepsilon^{\prime \prime} \beta$,only are derived from the $\varepsilon^{\prime \prime}{ }_{\alpha+\beta}$ values after subtracting the $\alpha$-contribution via a power law extrapolation to $v=1 \mathrm{kHz}$ on the high frequency flank, meant to indicate the loss spectrum in case the $\alpha$ process were suppressed.

MTHF forms stable glass states even for the lowest substrate temperatures, as indicated by loss ratios below unity for all deposition conditions of this study, see Fig. 2 and Fig. 5. However, propylbenzenes deposited below $0.60 T_{\mathrm{g}}$ show loss ratios above unity, i.e., $\chi^{\prime \prime} \mathrm{AD}>\chi^{\prime \prime}$ ann in Fig. 2 and Fig. 5. This can be interpreted as deposition at low substrate temperatures resulting in structures that are less dense than what is observed for the annealed state, leading to higher loss values in the asdeposited glass relative to the annealed state. Qualitatively, this picture would be consistent with the molar volumes of IPB and NPB rising above the level of the supercooled liquid for $T_{\mathrm{dep}}<0.8 T_{\mathrm{g}}$, which 
has been reported by Ishii et al. ${ }^{19,20,21,22}$ A likely cause for this behavior is the reduced surface mobility of propylbenzenes (relative to MTHF) based on intermolecular interactions via $\pi$-bonds of the aromatic phenyl rings, so called $\pi$ - $\pi$-interactions. This may lead to less stable and more open structures than observed for the annealed state, even for deposition conditions for which MTHF still shows $\chi^{\prime \prime} \mathrm{AD} / \chi^{\prime \prime}$ ann $<1$. These states with $\chi^{\prime \prime} \mathrm{AD}>\chi^{\prime \prime}$ ann for IPB and NPB may be associated with enthalpies that are higher than those of the annealed material, similar to the state expected after quenching the liquid very rapidly. By extrapolation of the data in Fig. 5, MTHF may enter this $\chi^{\prime \prime} \mathrm{AD}>\chi^{\prime \prime}$ ann regime for deposition temperatures $T_{\mathrm{dep}}<0.25 T_{\mathrm{g}}$. That MTHF reaches values for the $\chi^{\prime \prime} \mathrm{AD} / \chi^{\prime \prime}$ ann ratio that are lower than those of IPB or NPB is consistent with its higher dipole moment and thus larger secondary relaxation amplitude, $\Delta \varepsilon_{\beta}$, while the losses in glassy IPB and NPB have a higher 'near constant loss' (NCL) baseline contribution.

\section{B. Reduction of $\chi^{\prime}$ levels}

As has been reported earlier for MTHF, ${ }^{18}$ the storage component of the susceptibility, $\chi^{\prime}$, is reduced for as-deposited glasses relative to the annealed case, i.e., $\chi^{\prime} \mathrm{AD} / \chi^{\prime}$ ann $<1$, see Fig. 4 . This reduction by a factor of about two brings $\chi^{\prime}$ AD very close to the value of pure electronic polarizability effects, with $\varepsilon_{\infty}$ $\approx n^{2}$, where $n$ is the index of refraction. The present results for IPB and NPB establish that this PVD induced reduction of $\chi^{\prime}$ is not specific to MTHF, but possibly is a general feature of vapor-deposited films. The levels of $\chi^{\prime} \mathrm{AD} / \chi^{\prime}$ ann observed for IBP and NPB are near 0.9 , and thus much closer to unity than in the MTHF case. That the effect is small in the propylbenzenes relative to MTHF is due to the small values of $\Delta \varepsilon$ for IPB and NPB, so that eliminating all dipolar contributions has a much larger effect on the $\chi^{\prime}$ value for MTHF $\left(\Delta \varepsilon / \varepsilon_{\infty} \approx 5.3\right)$ than for IPB $\left(\Delta \varepsilon / \varepsilon_{\infty} \approx 0.12\right)$ and $\operatorname{NPB}\left(\Delta \varepsilon / \varepsilon_{\infty} \approx 0.10\right)$. The data for MTHF displays a slight but systematic increase in $\chi^{\prime}{ }_{\mathrm{AD}} / \chi^{\prime}$ ann with the film thickness $d,{ }^{18}$ an effect that is not resolved for the propylbenzenes, see Fig. 4a.

It may seem that this reduction of $\chi^{\prime}$ in as-deposited films is another manifestation of kinetic stability and enhanced density, but this is not the case. Unlike the situation with the suppression of $\chi^{\prime \prime} \mathrm{AD}$, the reduction of the level of $\chi^{\prime} \mathrm{AD}$ relative to the annealed case is not systematically dependent on the deposition temperature, see Fig. 4b. This indicates that the $\chi^{\prime} \mathrm{AD}$ effect is not directly linked to kinetic stability, but is rather still present for deposition conditions where stability is not observed. Another signature of the independence of $\chi^{\prime} \mathrm{AD} / \chi^{\prime}$ ann is the difference in the annealing behavior: while the kinetic stability of as-deposited films is erased at around $1.05 T_{\mathrm{g}}$ (see Fig. 1), $\chi^{\prime}$ AD remains reduced 
below $\chi^{\prime}$ ann for temperatures around $1.15 T_{\mathrm{g}}$ (see Fig. 3 and Fig. 4). Further evidence is required to elucidate the origin and persistence of these $\chi^{\prime}$ AD effects.

In the case of MTHF, a value of $\chi^{\prime} \mathrm{AD} / \chi^{\prime}$ ann $\approx 0.5$ is found for temperatures $T<T_{\mathrm{g}}$, and a similar ratio is observed for how far $\chi^{\prime}$ ann remains below the reference level $\chi^{\prime}$ sL,ref $=16.3$ for temperatures $T>T_{\mathrm{g}}$, see Fig. 3. For both propylbenzenes, $\chi^{\prime} \mathrm{AD}<\chi^{\prime}$ ann is also found for $T<T_{\mathrm{g}}$, but for IPB and NPB $\chi^{\prime}$ ann $=$ $\chi^{\prime}$ 'sL,ref for temperatures $T>T_{\mathrm{g}}$. This supports the previous notion that the reductions of $\chi^{\prime}$ in the glass and in the liquid are independent features. ${ }^{18}$ The values of $\chi^{\prime}$ in the liquid state above $T_{\mathrm{g}}$ are addressed in Section IV.C.

\section{Polyamorphism}

A particularly interesting feature of vapor-deposited MTHF is reflected in Fig. 3 in the range $T>$ $1.1 T_{\mathrm{g} .}{ }^{18}$ All initial temperature scans of the as-deposited films as well as the values obtained after annealing at $1.2 T_{\mathrm{g}}$ (black stars) fail to reach the susceptibility level of the bulk reference liquid (red crosses) at $\chi^{\prime}$ SL,ref $=16.3$. Instead, only about half of the static dielectric susceptibility is observed for MTHF films annealed at $1.2 T_{\mathrm{g}}$, even after the system has clearly entered a liquid state with considerable dipole mobility. As has been demonstrated earlier, the ordinary liquid state is recovered by a liquid-liquid transition at temperatures near $1.3 T_{\mathrm{g}}$, thus indicating polyamorphism accessed via PVD. ${ }^{18}$ The reduced $\chi^{\prime}$ value observed after annealing just above $T_{\mathrm{g}}$ could arise from a state associated with a lower Kirkwood correlation factor $g_{\mathrm{K}}$, but a heterogeneous mixture of the ordinary liquid and a non-polar phase would also explain these findings. ${ }^{18,39}$

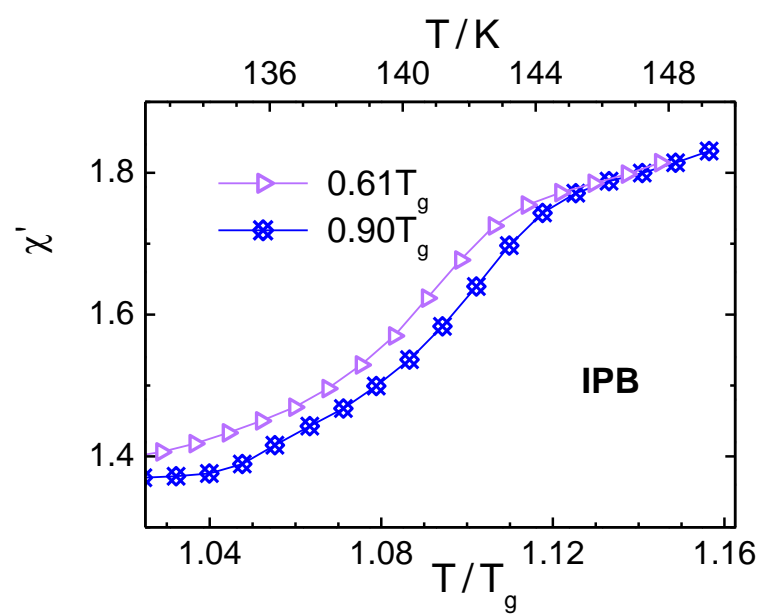

Fig. 7. Storage component of the susceptibility, $\chi^{\prime}$, of as-deposited IPB, plotted against reduced temperature, $T / T_{\mathrm{g}}$ for two distinct cases: $T_{\mathrm{dep}}=0.61 T_{\mathrm{g}}$ and $T_{\mathrm{dep}}=0.90 T_{\mathrm{g}}$. Selected data from Fig. 3 on expanded scales. 
Motivated by the curious light scattering reported by Ishii et al. for vapor-deposited benzene derivatives when heated above $T_{\mathrm{g}},{ }^{19}$ it is interesting to investigate whether propylbenzenes display a similar signature of polyamorphism in the liquid state. For IPB and NPB, the liquid phases with unusual scattering behavior appeared between $130 \mathrm{~K}$ and $140 \mathrm{~K}$, but only for films deposited onto low substrate temperatures, $T_{\text {dep }}<0.7 T_{\mathrm{g}}$, i.e., only when films of low stability were deposited. For both compounds, IPB and NPB, more ordinary liquid behavior was recovered prior to the onset of crystallization. ${ }^{19}$ Scattering implies a heterogeneous mix of different refractive indices, which could be visible when comparing the $\chi^{\prime}$ rise for IPB around $T=140 \mathrm{~K}=1.08 T_{\mathrm{g}}$ for two cases, $T_{\mathrm{dep}}<0.70 T_{\mathrm{g}}$ and $T_{\text {dep }}>0.85 T_{\mathrm{g}}$. Fig. 7 compares the rise of $\chi^{\prime}$ associated with the glass to liquid transition for the case of $T_{\text {dep }}=0.61 T_{\mathrm{g}}$ and $T_{\mathrm{dep}}=0.90 T_{\mathrm{g}}$, where only the $T_{\mathrm{dep}}=0.61 T_{\mathrm{g}}$ curve is expected to display unusual effects. Apart from a slight shift in the onset temperature that plausibly results from kinetic stability differences, no intermediate phase change is observed in Fig. 7 that differs visibly in terms of the dielectric constant, where we estimate that amplitude steps of about 0.04 would be resolved. A situation consistent with both observations could be that IPB enters a heterogeneous state that is a mixture of two structures with different refractive indices that leads to scattering, but is not resolved in a dielectric measurement of $\chi^{\prime}$ at $v=1 \mathrm{kHz}$.

The only conclusion that can be derived from the data in Fig. 7 is that the deposition temperature does not have an effect on the dielectric susceptibility that is analogous to what Ishii et al. have observed by optical techniques. While the deposition conditions and heating rates of the two studies are quite similar, the substrate material (gold versus borosilicate glass) and the detection technique differ considerably. Unless it is a matter of experimental conditions, the transformation process of stable glasses could play a role in the findings. Thick vapor-deposited films such as the ones used in the study of Ishii et al. ${ }^{19}$ are likely to transform to the ordinary state via a heterogeneous process, ${ }^{40,41}$ which should induce enhanced scattering within the temperature range of the transformation process.

\section{Summary and Conclusion}

In this work we report on the dielectric properties of glasses of two propylbenzenes (IPB, NPB) prepared by vapor deposition using a wide range of deposition temperatures, $0.3 T_{\mathrm{g}}<T_{\text {dep }}<1.1 T_{\mathrm{g}}$. Results for both the as-deposited and the annealed state are reported and compared with results obtained recently for 2-methyltetrahydrofuran (MTHF). All three materials show the typical signatures of kinetic stability that is most pronounced for $T_{\mathrm{dep}} \approx 0.85 T_{\mathrm{g}}$ : an onset temperature for the glass-toliquid transformation that is shifted to higher temperatures, and a reduced molecular mobility in the 
glass seen as smaller intensity of the $\beta$-relaxation. Another common feature is the reduction of $\chi^{\prime}$ in the as-deposited glasses, albeit a much more pronounced effect for MTHF due to the differences in the contributions of relaxation amplitudes $(\Delta \varepsilon)$ and high frequency dielectric constants $\left(\varepsilon_{\infty}\right)$ to $\chi^{\prime}\left(\Delta \varepsilon / \varepsilon_{\infty} \approx\right.$ 5.3 for MTHF, $\Delta \varepsilon^{\prime} \varepsilon_{\infty} \approx 0.12$ for IPB, and $\Delta \varepsilon / \varepsilon_{\infty} \approx 0.10$ for NPB). The magnitude of this effect is independent of how strongly the loss $\left(\chi^{\prime \prime}\right)$ is suppressed, so that the origin of this $\chi^{\prime}$ reduction is not obvious.

Films of IPB and NPB have been prepared by vapor deposition using parameters similar to those employed by Ishii et al. ${ }^{19}$ who reported unusual scattering effects for their films deposited below $0.7 T_{\mathrm{g}}$ and when heated across the 135 to $145 \mathrm{~K}$ range in case of IPB. The present study has not revealed concomitant changes in the dielectric behavior, i.e., featureless increases of $\chi^{\prime}(T)$ from the glass to the liquid level are observed, and the curves for $T_{\mathrm{dep}}=0.61 T_{\mathrm{g}}$ and $T_{\mathrm{dep}}=0.90 T_{\mathrm{g}}$ do not show qualitative differences. Possible explanations are residual discrepancies in the sample preparation or that the unusual state that scatters light effectively has practically the same dielectric properties as the ordinary liquid.

\section{Supplementary Material}

See Supplementary Material for plots of reference data, data collected at further deposition temperatures for MTHF and IPB, as well as Propylbenzene data before smoothing by sliding averages.

\section{Acknowledgments}

This work was supported by the National Science Foundation under Grant No. CHE-1564663 and CHE- 1854930. B.R. is grateful for support from the VILLUM Foundation's Matter grant (16515).

\section{References}

1 S. F. Swallen, K. L. Kearns, M. K. Mapes, Y. S. Kim, R. J. McMahon, M. D. Ediger, L. Yu, and S. Satija, Science 315, 353 (2007).

2 D. Yokoyama, A. Sakaguchi, M. Suzuki, and C. Adachi, Org. Electron. 10, 127 (2009).

3 E. Leon-Gutierrez, A. Sepúlveda, G. Garcia, M. T. Clavaguera-Mora, and J. Rodríguez-Viejo, Phys. Chem. Chem. Phys. 12, 14693 (2010).

4 S. Léonard and P. Harrowell, J. Chem. Phys. 133, 244502 (2010). 
5 S. L. L. M. Ramos, M. Oguni, K. Ishii, and H. Nakayama, J. Phys. Chem. B 115, 14327 (2011).

6 T. Liu, K. Cheng, E. Salami-Ranjbaran, F. Gao, C. Li, X. Tong, Y.-C. Lin, Y. Zhang, W. Zhang, L. Klinge, P. J. Walsh, and Z. Fakhraai, J. Chem. Phys. 143, 084506 (2015).

7 D. Bhattacharya and V. Sadtchenko, J. Chem. Phys. 141, 094502 (2014).

8 L. Berthier, P. Charbonneau, E. Flenner, and F. Zamponi, Phys. Rev. Lett. 119, 188002 (2017).

9 L. W. Antony, N. E. Jackson, I. Lyubimov, V. Vishwanath, M. D. Ediger, and J. J. de Pablo, ACS Cent. Sci. 3, 415 (2017).

10 M. D. Ediger, J. Chem. Phys. 147, 210901 (2017).

11 K. L. Kearns, S. F. Swallen, M. D. Ediger, T. Wu, Y. Sun, and L. Yu, J. Phys. Chem. B 112, 4934 (2008).

12 M. S. Beasley, C. Bishop, B. J. Kasting, and M. D. Ediger, J. Phys. Chem. Lett. 10, 4069 (2019).

13 L. Yu, Adv. Drug Deliv. Rev. 100, 3 (2016).

14 M. Tylinski, Y. Z. Chua, M. S. Beasley, C. Schick, and M. D. Ediger, J. Chem. Phys. 145, 174506 (2016).

15 M. Tylinski, M. S. Beasley, Y. Z. Chua, C. Schick, and M. D. Ediger, J. Chem. Phys. 146, 203317 (2017).

16 S. Capponi, S. Napolitano, and M. Wübbenhorst, Nat. Comm. 3, 1233 (2012).

17 H.-B. Yu, M. Tylinski, A. Guiseppi-Elie, M. D. Ediger, and R. Richert, Phys. Rev. Lett. 115, 185501 (2015).

18 B. Riechers, A. Guiseppi-Elie, M. D. Ediger, and R. Richert, J. Chem. Phys. 150, 214502 (2019).

19 K. Ishii, H. Nakayama, R. Moriyama, and Y. Yokoyama, Bull. Chem. Soc. Jpn. 82, 1240 (2009).

20 K. Ishii, H. Nakayama, T. Okamura, M. Yamamoto, and T. Hosokawa, J. Phys. Chem. B 107, 876 (2003).

21 K. Ishii, H. Nakayama, S. Hirabayashi, and R. Moriyama, Chem. Phys. Lett. 459, 109 (2008).

22 K. Ishii, H. Nakayama, and R. Moriyama, J. Phys. Chem. B 116, 935 (2012).

23 L. Yang, A. Guiseppi-Wilson, and A. Guiseppi-Elie, Biomed. Microdevices 13, 279 (2011). 
24 A. I. Nielsen, T. Christensen, B. Jakobsen, K. Niss, N. B. Olsen, R. Richert, and J. C. Dyre, J. Chem. Phys. 130, 154508 (2009).

25 W. Olthuis, W. Streekstra, and P. Berveld, Sensors and Actuators B: Chemical 24, 252 (1995).

26 M. Mizukami, H. Fujimori, and M. Oguni, Prog. Theor. Phys. Suppl. 126, 79 (1997).

27 R. Richert and C. A. Angell, J. Chem. Phys. 108, 9016 (1998).

28 A. Laventure, A. Gujral, O. Lebel, C. Pellerin, and M. D. Ediger, J. Phys. Chem. B 121, 2350 (2017).

29 A. Sepulveda, M. Tylinski, A. Guiseppi-Elie, R. Richert, and M. D. Ediger, Phys. Rev. Lett. 113, 045901 (2014).

30 C. Rodríguez-Tinoco, K. L. Ngai, M. Rams-Baron, J. Rodríguez-Viejo, and M. Paluch, Phys. Chem. Chem. Phys. 20, 21925 (2018).

31 K. R. Whitaker, M. Tylinski, M. Ahrenberg, C. Schick, and M. D. Ediger, J. Chem. Phys. 143, 084511 (2015).

32 Y. Z. Chua, M. Ahrenberg, M. Tylinski, M. D. Ediger, and C. Schick, J. Chem. Phys. 142, 054506 (2015).

33 H.-B. Yu, Y. Luo, and K. Samwer, Adv. Mater. 25, 5904 (2013).

34 J. Q. Wang, Y. Shen, J. H. Perepezko, and M. D. Ediger, Acta Mater. 104, 25 (2016).

35 C. Rodríguez-Tinoco, M. Rams-Baron, K. L. Ngai, K. Jurkiewicz, J. Rodríguez-Viejo, and M. Paluch, Phys. Chem. Chem. Phys. 20, 3939 (2018).

36 M. Vogel and E. Rössler, J. Chem. Phys. 114, 5802 (2001).

37 M. Vogel and E. Rössler, J. Chem. Phys. 115, 10883 (2001).

38 B. J. Kasting, M. S. Beasley, A. Guiseppi-Elie, R. Richert, M. D. Ediger, J. Chem. Phys. (submitted).

39 A. Kasina, T. Putzeys, and M. Wübbenhorst, J. Chem. Phys 143, 244504 (2015).

40 K. L. Kearns, M. D. Ediger, H. Huth, and C. Schick, J. Phys. Chem. Lett. 1, 388 (2010).

41 J. Ràfols-Ribé, A. Vila-Costa, C. Rodríguez-Tinoco, A. F. Lopeandía, J. Rodríguez-Viejo, and M. 
Gonzalez-Silveira, Phys. Chem. Chem. Phys. 20, 29989 (2018). 


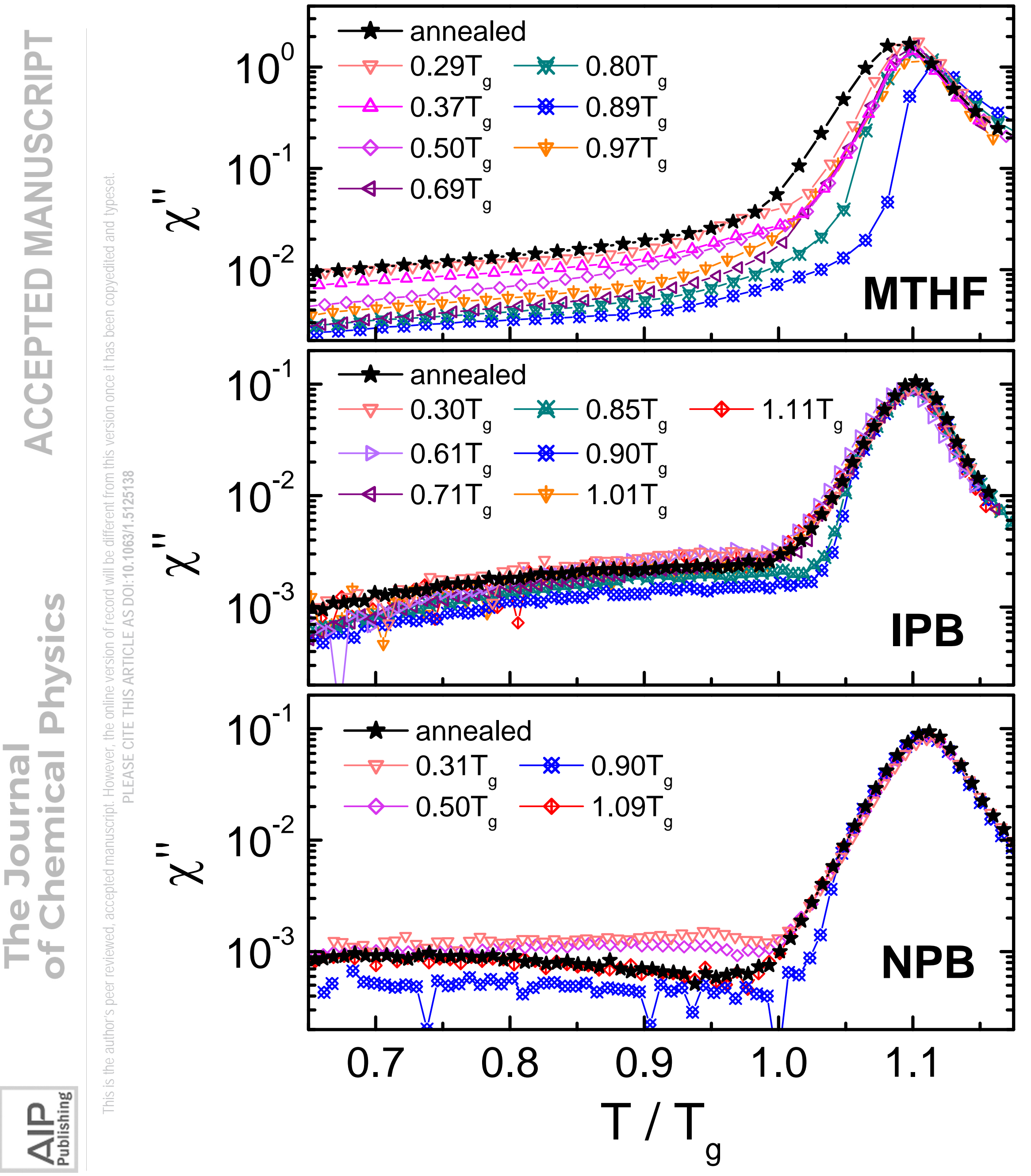




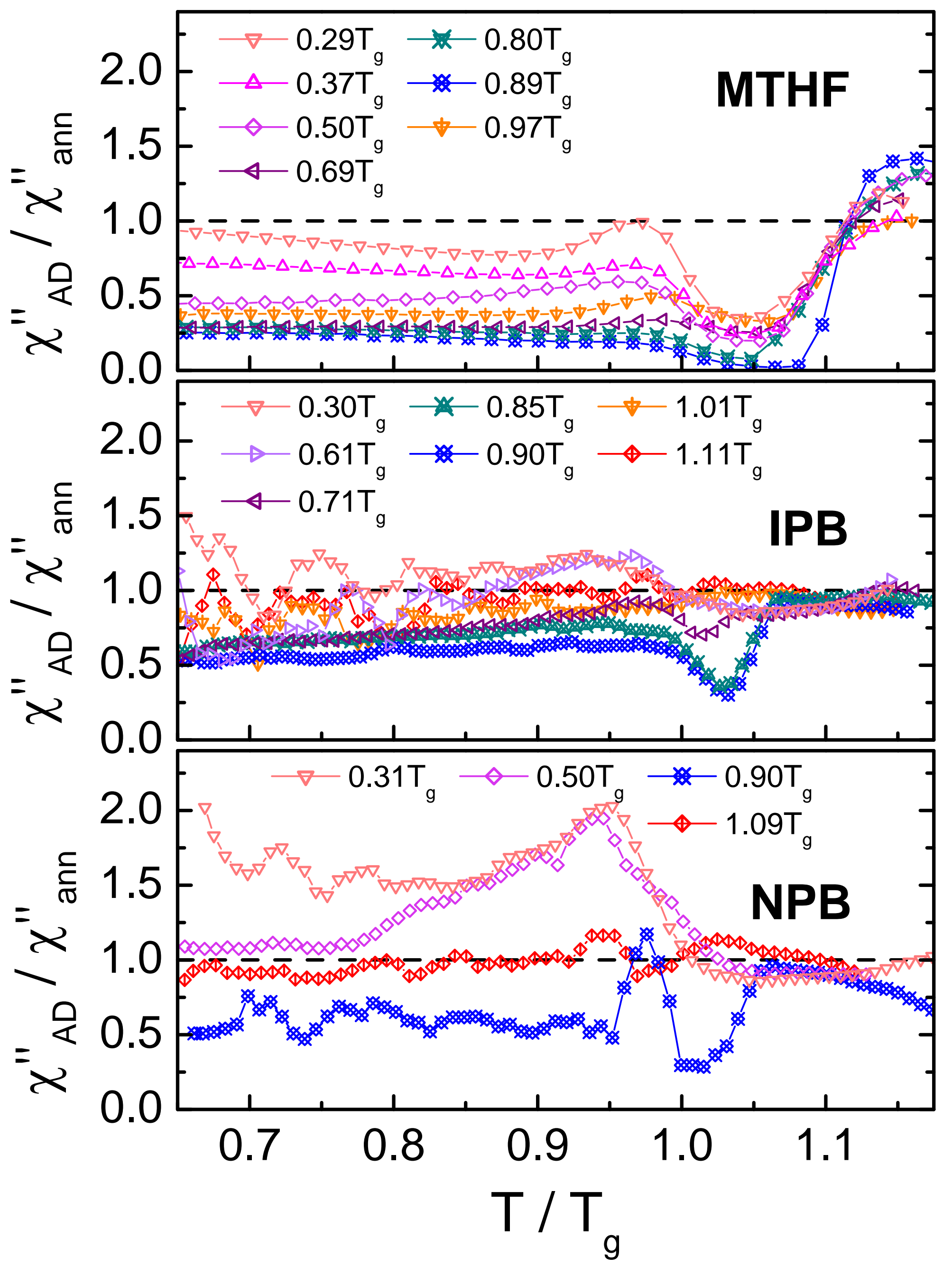




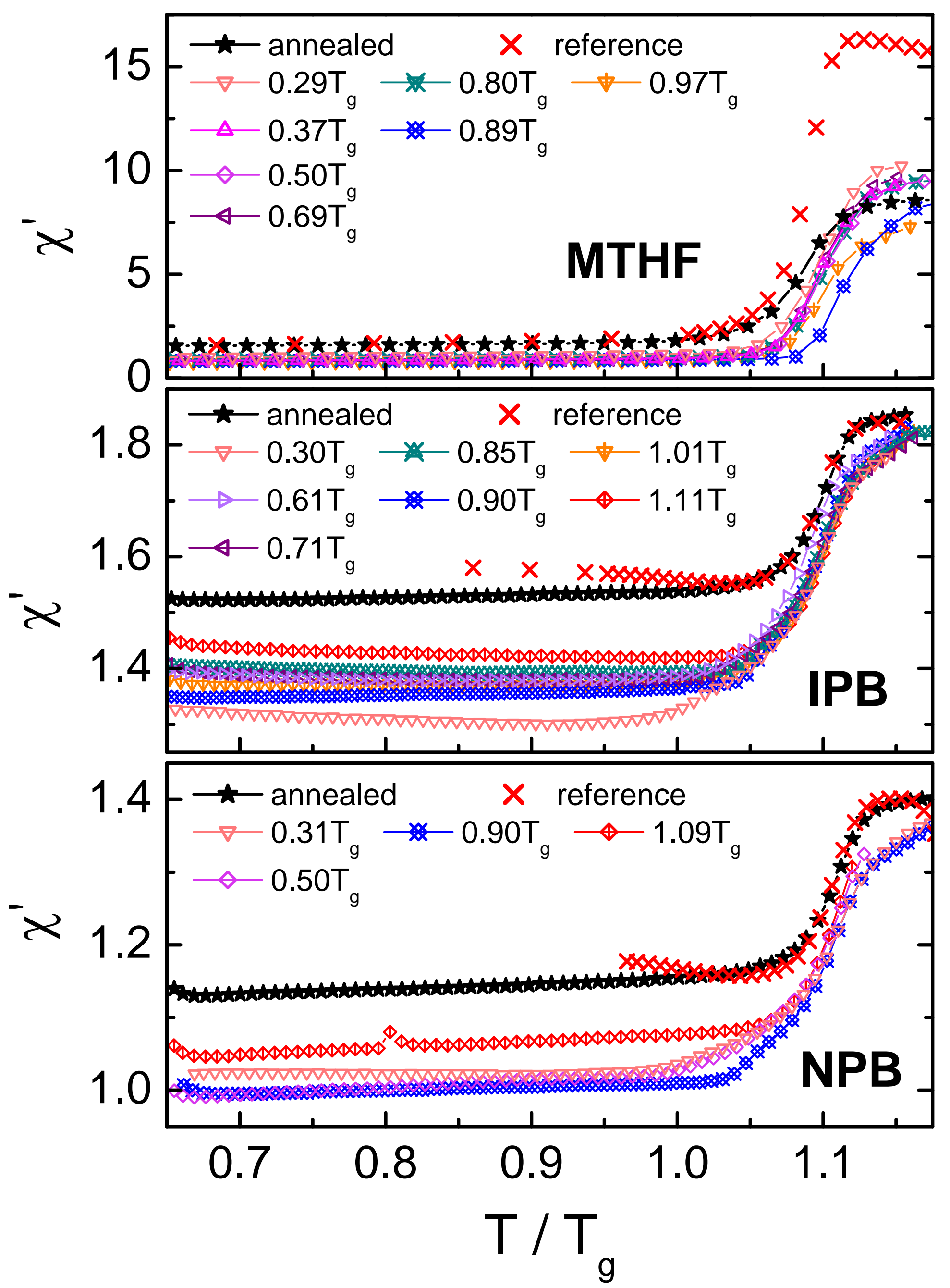



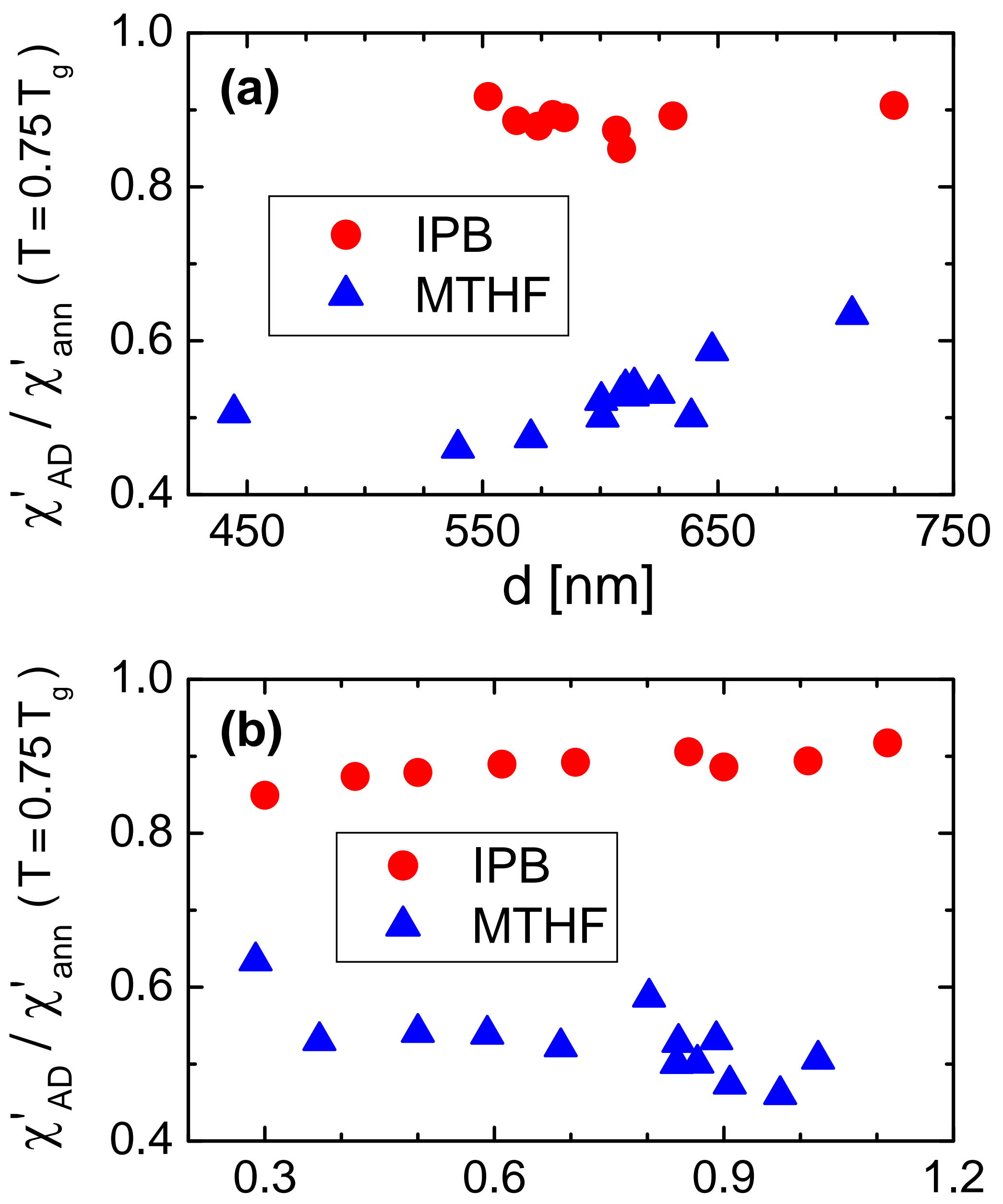

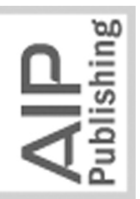

$$
\mathrm{T}_{\text {dep }} / \mathrm{T}_{\mathrm{g}}
$$




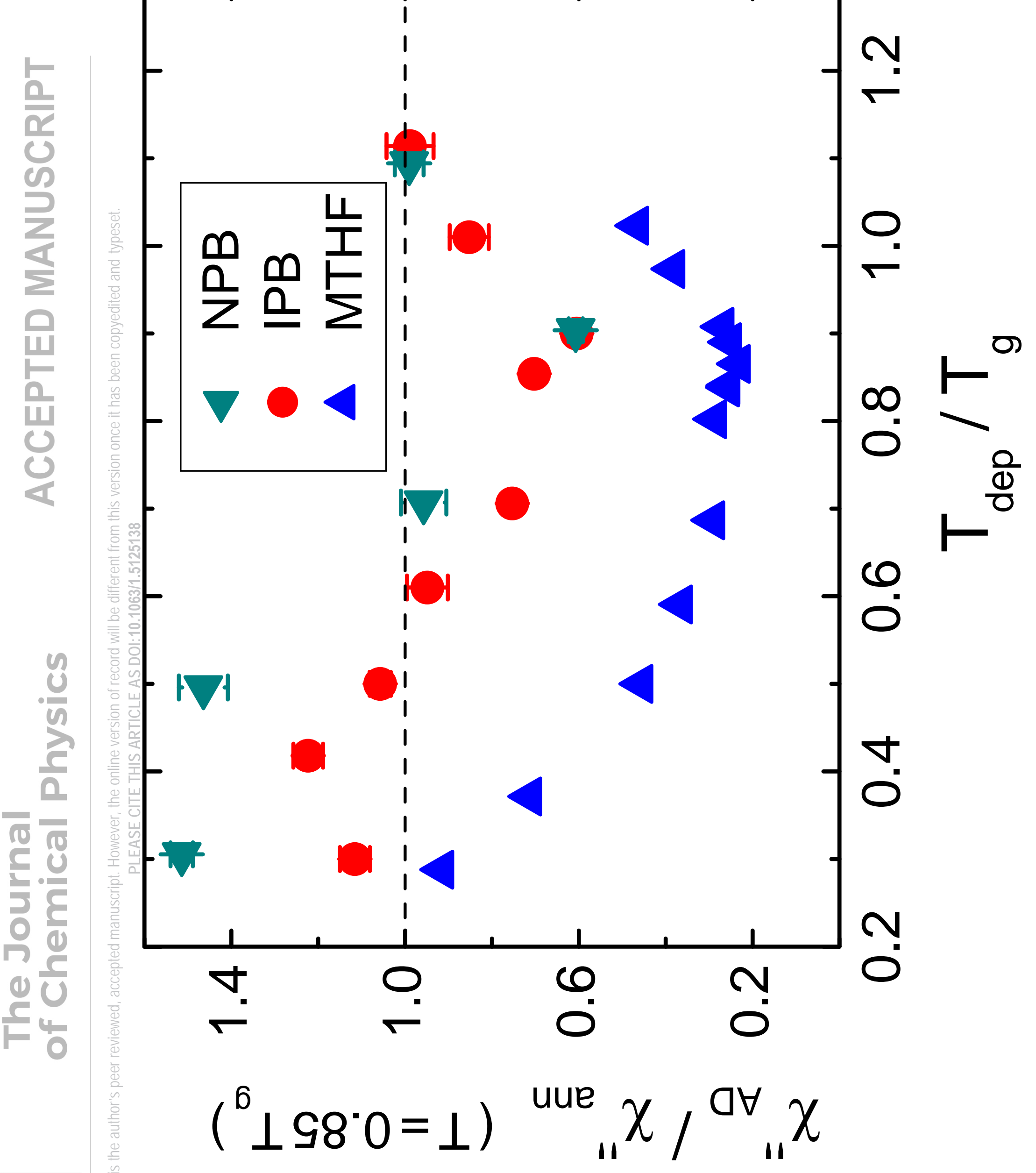

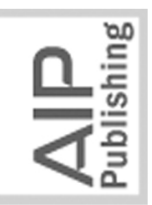




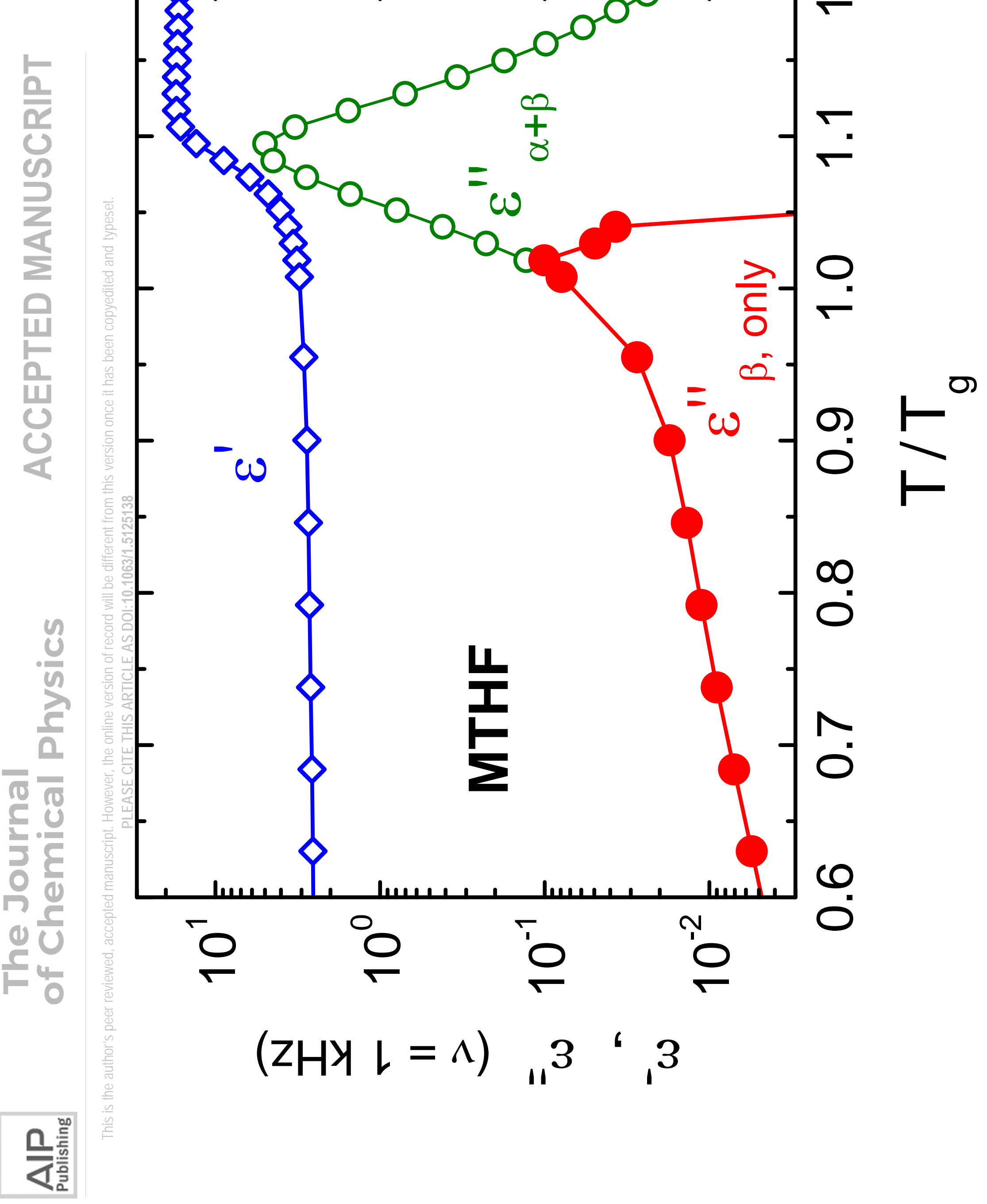


